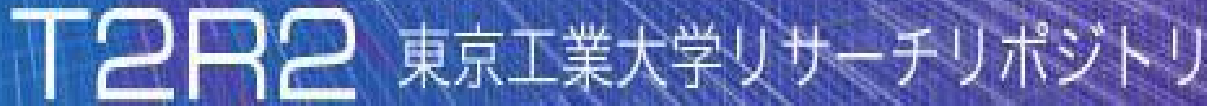

\section{Tokyo Tech Research Repository}

\section{論文 /著書情報 \\ Article /Book Information}

\begin{tabular}{|c|c|}
\hline Title & Soft-Amphibious Robot using Thin and Soft McKibben Actuator \\
\hline Authors & A.A.M.Faudzi, M. R. M. Razif, G. Endo, H. Nabae, K. Suzumori \\
\hline Citation & $\begin{array}{l}\text { Proceedings of the } 2017 \text { IEEE /ASME International Conference on } \\
\text { Advanced Intelligent Mechatronics (AIM 2017), pp. 981-986 }\end{array}$ \\
\hline Pub. date & 2017,7 \\
\hline DOI & http://dx.doi.org/10.1109/AIM.2017.8014146 \\
\hline URL & http://www.ieee.org/ndex.html \\
\hline Copyright & $\begin{array}{l}\text { (c) } 2017 \text { IEEE. Personal use of this material is permitted. Permission } \\
\text { from IEEE must be obtained for all other uses, in any current or future } \\
\text { media, including reprinting/republishing this material for advertising or } \\
\text { promotional purposes, creating new collective works, for resale or } \\
\text { redistribution to servers or lists, or reuse of any copyrighted component } \\
\text { of this work in other works. }\end{array}$ \\
\hline Note & This file is author (final) version. \\
\hline
\end{tabular}




\title{
Soft-Amphibious Robot using Thin and Soft McKibben Actuator
}

\author{
A. A. M. Faudzi, Member, IEEE, M. R. M. Razif, G. Endo, H. Nabae, K. Suzumori, Member, IEEE
}

\begin{abstract}
This paper introduces a quadruped soft-amphibious robot using $4.0 \mathrm{~mm}$ diameter thin and soft McKibben actuator. The robot utilizes its leg and body bending mechanism to locomote. For each leg, three links of the actuators are arranged in parallel with fixed upper and bottom part. Then, four actuators are arranged in parallel and fixed with a thin plastic plate in between the actuators for the body motion. The elastic deformation of the plastic plate actuated by the actuators assist in the side-to-side motion of the robot mimicking the gait of the biological creature like lizard/salamander during walking motion. FEM simulation studies were performed in Marc Mentat ${ }^{\circledR}$ to evaluate the bending behaviors and validated with an experimental test. Walking experiment was tested on a flat surface and on sand using two different gaits of trot and crawl gait. On the other hand, swimming experiment was tested inside water using only crawl gait. Different input pressure and frequency were varied to study the walking behavior. The robot successfully walks on a flat and $10^{\circ}$ incline plane with a maximum speed of $0.056 \mathrm{~m} / \mathrm{s}$ using trot gait and robust to move on sand and in water using the crawl gait at $0.041 \mathrm{~m} / \mathrm{s}$ and $0.022 \mathrm{~m} / \mathrm{s}$, respectively.
\end{abstract}

\section{INTRODUCTION}

Crawling and walking creatures have attract the attention of researchers because of their ability to move in numerous conditions including uneven terrain [1], incline or decline planes [2]. The study on kinematic and dynamic design of those biological creatures could assist humans in various applications such as pipe inspection [3] and many others. Such example of developed crawled robots were based on caterpillar [4], inchworm/earthworm [5-6], and lizard/salamander [7-8]. Some of the robots were controlled using four [9-10] and six [11-12] legs to mimic the crawling or walking motion of biological creatures. According to Parasuraman et al. [8], the quad legged robot will have a balance body in its walking motion by arranging proper sequence of gait.

In recent years, the lizard in particular, has been referred to as a biological model to locomote a mobile robot because of its advantage in multi-directional crawling with up to 24 degrees

*This work is supported by JSPS KAKENHI Grant Number 26249028

A.A.M. Faudzi is with the Centre of Artificial Intelligence and Robotics (CAIRO), Universiti Teknologi Malaysia, 81310 UTM Johor Bahru, Malaysia. (e-mail: athif(at)utm.my)

M. R. M. Razif is with Department of Electrical Engineering Technology, Faculty of Engineering Technology, Universiti Tun Hussein Onn Malaysia (UTHM), 86400 Parit Raja, Batu Pahat, Malaysia. (e-mail: rusydi(at)uthm.edu.my)

A. A. M. Faudzi, G. Endo, H. Nabae and K. Suzumori, are with the Department of Mechanical Engineering, Tokyo Institute of Technology 2-12-1, Ookayama, Meguro-ku, Tokyo 152-8552, Japan (e-mail: athif.f.aa, gendo.g.aa, nabae.h.aa, suzumori.k.aa(at)m.titech.ac.jp)

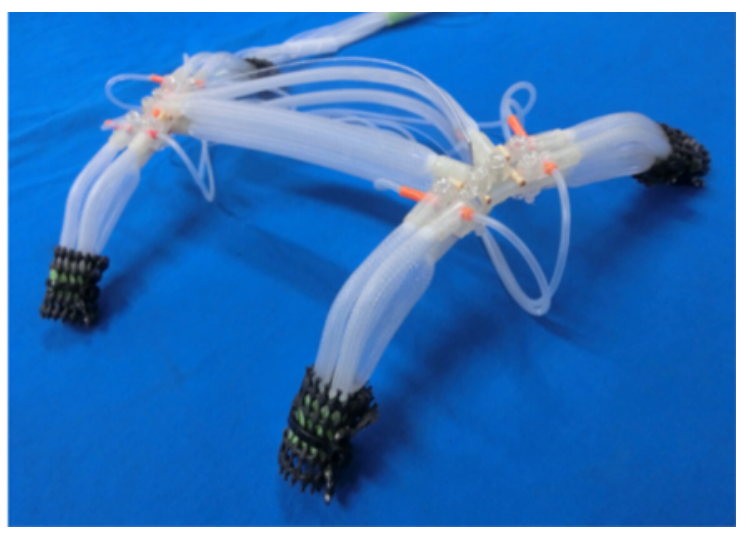

Figure 1. Soft-Amphibious robot

of freedom [13] including climbing a vertical walls. For stable walking in straight, curved and inclined/declined path, the positions of supporting feet and position of center of gravity play an important role [14]. For fast movement and turning curvature of turning motion, parameters such as body-limb coordination, frequency and amplitude phase lag of body oscillation are required [7]. They locomote with the feet lateral to the hip and shoulder joint. The position of the feet lateral to the body makes the bending of the body to the left move the right front foot forward relative to the left front foot, and the left back foot forward relative to the right back foot. Bending to the right has the reverse effect. Lizards take advantage of this motion to extend their steps by bending their backs from side to side as they locomote (walk or run) quadrupedal [15].

On the other hand, Urodela, an animal commonly known as Salamander, belongs to the amphibian group that is characterized by lizard-like appearance. Instead of climbing, salamander spends most of its time on ground and underwater. The salamander is a four-legged amphibian that is able to swim and walk, and is an excellent model to investigate vertebrate locomotion during its transition from aquatic to terrestrial environments [7].

Inspired by the flexible locomotion and amphibian capability of the salamander, this paper proposed a soft-amphibious robot as shown in Fig. 1. The robot is able to locomote on ground and in wet environment with simple body structure. The robot has four short legs lateral to its long body, light in weight and actuated by combination of 16 links of thin and soft McKibben actuators. The soft actuator has been used because of its simple structure, water resistance and suitable for watery environment, high compliance, light-weight, and flexible movement [16]. This robot utilizes two bending characteristics for its leg and body motions. Prior works have shown that the soft actuator could provide bending motion by manipulating the pattern of braided angle along the soft actuator cylindrical body [5]. Iwata et al. [17] combined two or more actuators into one. Another technique that could be 
used is combining two different patterns of braided angle in a single soft actuator cylindrical body [18] using multiple chambers actuator [19], or designing asymmetrical shape of the actuator as bellow shape [20].

In this paper, the soft-amphibious robot used the combination of thin soft McKibben actuator with the contraction type [21] to produce bending motion. By pressurizing one of the actuators with pneumatic pressure, the legs and the body of the robot could provide the bending motion. The combination of multi-actuators bending motion of the leg and body through the crawl gait provides flexible locomotion to produce forward or backward motion. The study contributes to our knowledge by presenting the bending characteristics (body and leg) by using the contraction of 4.0 $\mathrm{mm}$ thin soft McKibben actuator for the soft-amphibious robot locomotion. The remainder of the paper is organized as follows. In section II, the robot structure and specification is presented. Section III discusses two bending characteristics of thin soft McKibben actuator used for the soft-amphibious robot. Section IV discusses the circuitry, trot and crawl gait design and basic walking experiment using both gaits. The paper concludes with a brief appraisal and future work in section V.

\section{ROBOT STRUCTURE}

Salamander uses sprawling posture to support its body. The upper limbs are typically held horizontally, while the lower limbs are vertical. To closely mimic the leg structure, our soft-amphibious robot has a simplified design structure with four legs and a soft body as shown in Fig. 2. The soft body is supported by a plastic plate $(175 \times 25 \times 1 \mathrm{~mm})$, which acts as the support element for the robot and to drive and support the mechanism for body bending function. The robot mainly consists of a 16-link thin soft actuator that was assembled using non-metal screws making it light in weigh. The actuator cap was fabricated using 3D printer with ABS material. 12 links McKibben actuator were used for the leg part with three actuators for each leg and four links actuators for the body with two actuators at each side of the plastic plate. Each leg has one degrees of freedom (DoF) of roll motion using the bending properties. The leg has possibility to move in six directions, however in this report we used only three directions for the leg motion. The combination of body motion and one DoF leg movement is able to mimic the lizard/salamander locomotion.

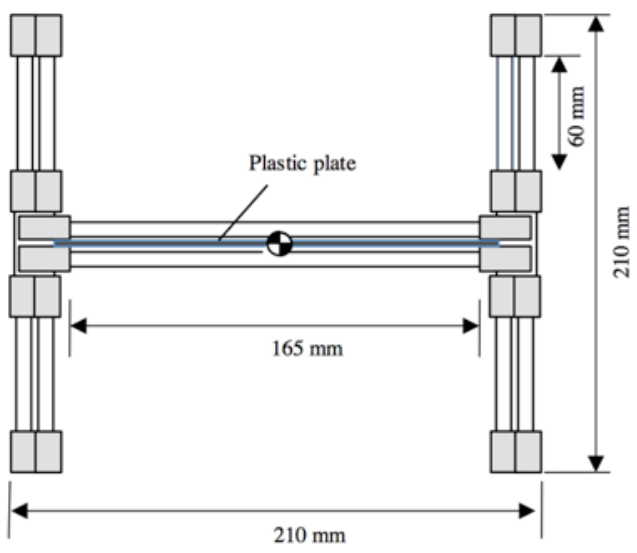

Figure 2. Schematic of robot design from top view
As the robot is very light and the cap of the actuator that acts as the foot is frictionless to the surface, we attached a rubber cloth around the leg to increase friction coefficient. Adding rubber shoes or heavier mechanism for the foot will add mass to the leg and significantly affects the inertia of the leg. Table I shows the specification of the soft-amphibious robot. Table II shows the length of soft actuator used for both leg and body motion.

TABLE I. SPECIFICATION OF SOFT-AMPHIBIOUS RoBOT

\begin{tabular}{|l|l|}
\hline \multicolumn{1}{|c|}{ Specification } & \multicolumn{1}{|c|}{ Parameters } \\
\hline Height & $50 \mathrm{~mm}$ \\
\hline Width & $210 \mathrm{~mm}$ \\
\hline Length & $210 \mathrm{~mm}$ \\
\hline Weight & $70 \mathrm{~g}$ \\
\hline
\end{tabular}

TABLE II. SPECIFICATION OF ACTUATORS USED

\begin{tabular}{|l|l|l|}
\hline \multirow{2}{*}{ Actuators } & \multicolumn{2}{|c|}{ Characteristics } \\
\cline { 2 - 3 } & \multicolumn{1}{|c|}{ Function } & Initial length \\
\hline Actuator 1 x (12) & leg & $60 \mathrm{~mm}$ \\
\hline Actuator 2 x (4) & body & $165 \mathrm{~mm}$ \\
\hline
\end{tabular}

\section{BENDING ThIn Soft McKibBEn ACtuAtor}

The McKibben actuator, also known as braided soft actuator, was initially developed by Schulte in 1960s [22]. The McKibben actuator consists of cylindrical rubber body and braided with fiber along its body. The function of braided fiber is to restrict the actuator expansion in radial direction, thus providing contraction, expansion or stiffness change. According to Trivedi et al. [23], the actuator would not contract nor extend at braided angle $55^{\circ}$, even when high pneumatic pressure is applied. The contraction would occur when the braided angle is less than $55^{\circ}$ and extend when braided angle is more than $55^{\circ}$.

We previously developed thin soft McKibben muscles that are light, small, and suitable for simple system [21]. The outer diameter of the silicone rubber tube is $4.0 \mathrm{~mm}$ with $1 \mathrm{~mm}$ thickness. The silicone tube is covered by 48 fibers of 0.22 $\mathrm{mm}$ Tetron monofilament along its cylindrical body surface to restrict the tube in radial expansion. The fiber angle was set to be $18^{\circ}$ for optimum contraction function of the actuator.

We simulate a single actuator in finite element software, Marc Mentat ${ }^{\circledR}$, to measure the contraction displacement when pneumatic pressure is supplied to the actuator. The material of the body and braid are set similar to the $4.0 \mathrm{~mm} \mathrm{McKibben}$ actuator with silicone rubber and Tetron, respectively. Fig. 3 shows the actuator before and after pressurized at $0.2 \mathrm{MPa}$. From there, we validate the simulated contraction behavior with the experimental result. Fig. 4 shows linear relation of the simulated contraction ratio and comparison with experimental data (with hysteresis) at different input pressure. The experimented contraction force is also shown in the figure. The experimental contraction ratio increased exponentially from 0.1 MPa and 0.2 $\mathrm{MPa}$ and gives around $22.5 \%$ and $43 \mathrm{~N}$ of contraction ratio and contraction force respectively, at 0.3 
$\mathrm{MPa}$ input pressure. As the operating pressure of the soft-amphibious robot is tested in between 0.15 to $0.3 \mathrm{MPa}$, the comparison between simulations and experimental is acceptable. Differences of contraction ratio in simulation and experiment are due to non-linear property of the rubber materials. Both simulation and experimental data was used to study the bending properties of the thin soft actuator as described in leg and body bending properties section.

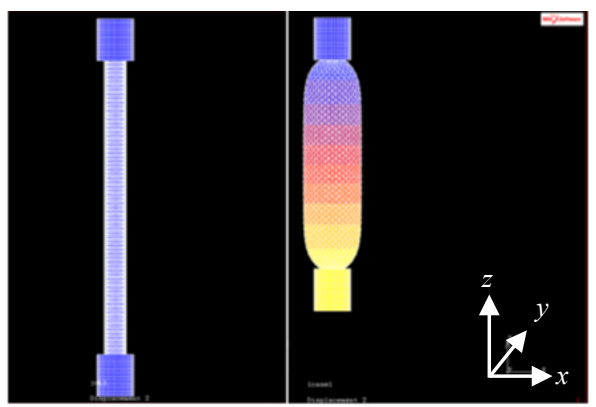

Figure 3. (Left) the actuator before pressurize (right) after presurrized with $0.2 \mathrm{MPa}$

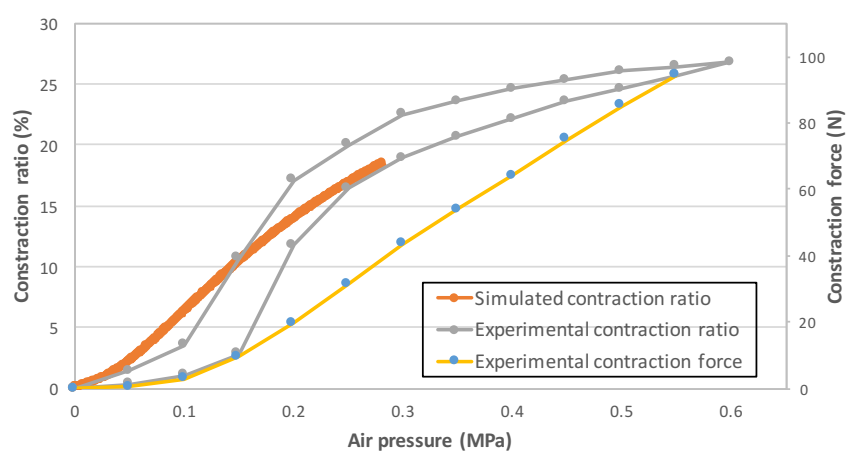

Figure 4. Comparison between simulated and experimental contraction ratio and contraction force at different air pressure

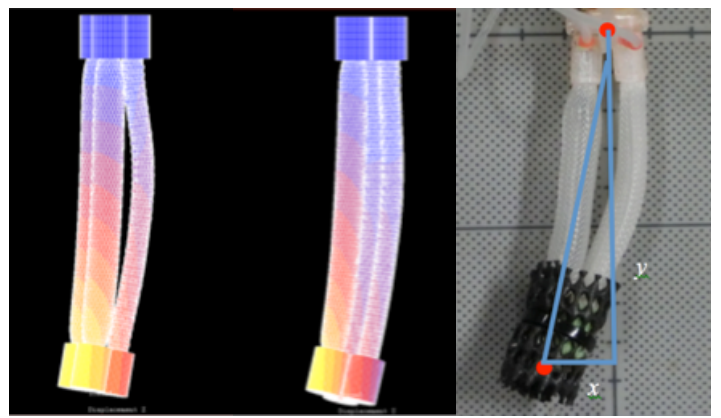

Figure 5. Soft-amphibious leg bends when pressurized at pressure 0.15 $\mathrm{MPa}$. (left) single actuator (middle) two actuators (right) experimental

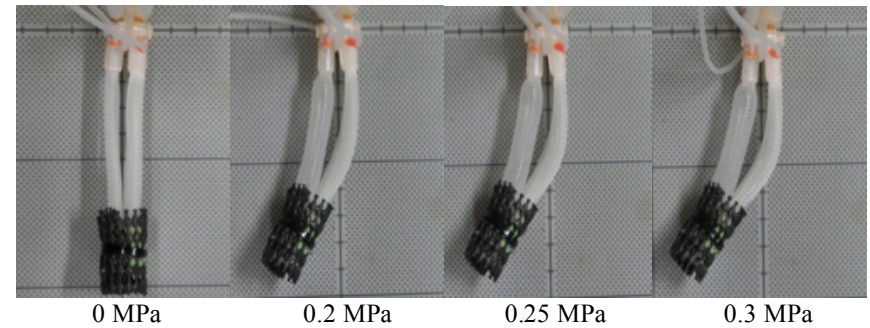

Figure 6. Experimental evaluation of leg bending for $0 \mathrm{MPa}, 0.2 \mathrm{MPa}$, $0.25 \mathrm{MPa}$ and $0.3 \mathrm{MPa}$

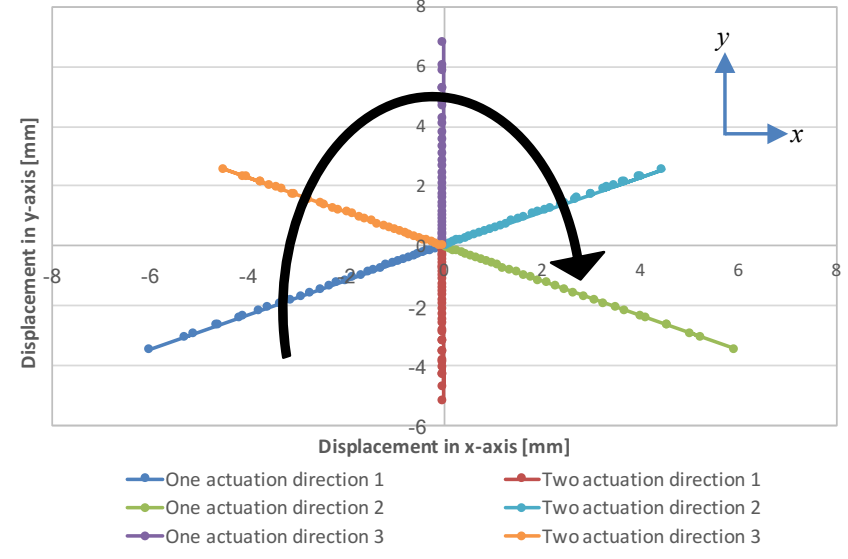

Figure 7. Simulation trajectory in $x$-axis and $y$-axis from $z$-axis view of leg bending at $0.15 \mathrm{MPa}$

\section{A. Leg bending property}

After simulating single thin McKibben actuator, the identical three thin McKibben actuators are arranged in parallel and attached together at the top and bottom. Pressure is then applied to one actuator and two actuators, respectively to study the bending motion as shown in Fig. 5. From the simulation, the bending performances occur towards the direction of pressurized actuator. Fig. 5 (right) shows the experimental test at $0.15 \mathrm{MPa}$ to validate the simulation data, which gives similar angle of $8^{\circ}$. The bending angle for the leg motion is calculated using Equation (1),

$$
\tan \theta=x / y
$$

where $x$ and $y$ represent the width and height, respectively.

Fig. 6 shows the experimental evaluation of bending motion with single actuator at $0 \mathrm{MPa}, 0.15 \mathrm{MPa}, 0.25 \mathrm{MPa}$ and $0.3 \mathrm{MPa}$ respectively. The usage of three actuators successfully provide three bending directions with the trajectory angle between $x$-axis and $y$-axis as shown in Fig. 7. Another three directional bending motions are also possible to be performed by pressurizing two actuators at the same time, making a total of six possible directional motions. However, the result shows actuating single actuator gives bigger bending angle thus only one actuation is selected for the soft-amphibious leg motion. By pressurizing each actuator at a time with certain delay, circular motion of leg (black arrow) can be obtained to assist robot locomotion using crawl gait as shown in Fig. 7. We did not manage to compare the simulated result of increased pressure value (more than $0.15 \mathrm{MPa}$ ) with the experimental result due to computational limits. The bending angles of leg at each input pressure are plotted in Fig. 10.

\section{B. Body bending property}

Body bending property of the soft-amphibious could help the robot to mimic the salamander locomotion. The bending motion is performed with the assistance of a plastic plate that is placed in between two actuators at each side of the plate. This bending property utilizes the plastic plate motion, which deforms elastically when left and right actuators are actuated. 
The aptitude of the plastic in the middle of soft-amphibious body to contribute to body bending has been validated by FEM simulation. Fig. 8 shows the bending angle provided by the body structure with and without appearance of plastic before and after pressurized with $0.2 \mathrm{MPa}$. The bending angle of the body increases as the input pressure increased. This is due to the bigger amount of contraction force produced by the McKibben actuator. The allowable load, $F$ is calculated through Equation (2) where the force of McKibben actuator should be within this value.

$$
F=n \pi^{2} E I / L^{2}
$$

where $n$ is the factor accounting for the end conditions, we consider one end fixed, one end free with $n=0.25, E$ is the modulus of elasticity of Polypropylene, $E=2 \mathrm{GPa}, I$ is the moment of inertia, $I=1.302 \times 10^{-9} \mathrm{~m}^{4}$, and $L$ is the length of the plastic plate with $L=175 \mathrm{~mm}$. The calculated allowable load of force, $F$ is $210 \mathrm{~N}$. In the body bending structure, we applied two links of soft actuator on each side. As single actuator can produce contraction force of $43 \mathrm{~N}$, the overall operated force at $0.3 \mathrm{MPa}$ is only $86 \mathrm{~N}$ which is within the allowable load of force of the plastic structure. The angle of the bending could be calculated using deflection Equation (3).

$$
\theta=F_{m} \sin \alpha \cdot L^{2} / 2 E I
$$

The side-to-side deformation of the plastic plate also improves the motion for the soft-amphibious robot to move similar to the lizard/salamander locomotion. Bending of the body to the right moves the left front foot forward relative to the right front foot, and the right back foot forward relative to the left back foot as shown in Fig. 9. Bending to the left has the reverse effect. Bending the body side to side will help the salamander to extend their steps as they locomote.

Fig. 9 also shows the experimental evaluation of body bending at initial condition $0 \mathrm{MPa}, 0.2 \mathrm{MPa}, 0.25 \mathrm{MPa}$ and 0.3 $\mathrm{MPa}$. From the figure, we can observe that the higher the input pressure, the higher the bending of the robot body angle of the robot. Bending of both leg and body properties are shown in Fig. 10 at different pressure from $0.1 \mathrm{MPa}$ to $0.3 \mathrm{MPa}$. From the figure, we identified the body bending angle and leg bending angle of $18.8^{\circ}$ and $16^{\circ}$ respectively, at $0.3 \mathrm{MPa}$ input pressure. The leg-bending angle of $16^{\circ}$ enables the robot to stand with enough height from the ground to ease the locomotion.

\section{GAIT DESIGN AND WALKING EXPERIMENT}

\section{A. Overall System}

The robot is controlled using Arduino Mega 2560. The system is powered by two sets of $6 \mathrm{~V}$ DC Li-On battery. DC-DC converter is used to convert to $24 \mathrm{~V}$ to drive 16 Koganei B005E1-PS on/off valves. In the present design, the compressed air is supplied by an air compressor which is not on board.

\section{B. Trot and crawl gait}

Walking gait for quadruped such as turtles uses tripod gait and achieve its maximum stability by keeping the center of gravity within the tripods of three feet on the ground. However, salamander they walk using trot gait and swim using anguilliform gait. For this robot, the trot gait considers two legs as minimum number of legs to support the robot

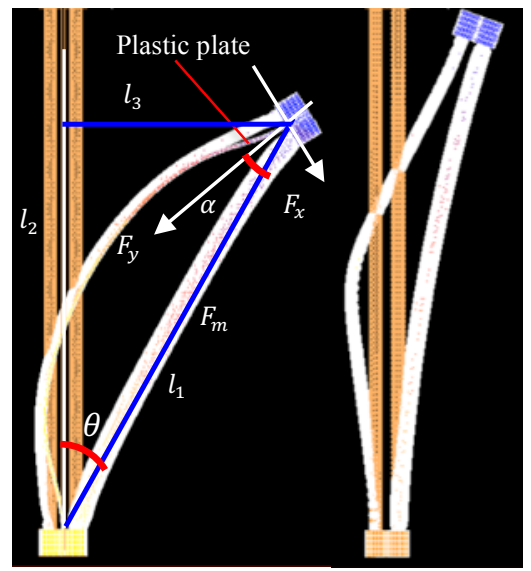

Figure 8. Simulated body bending at $0.2 \mathrm{MPa}$ input pressure with plastic(left) and without plastic (right)

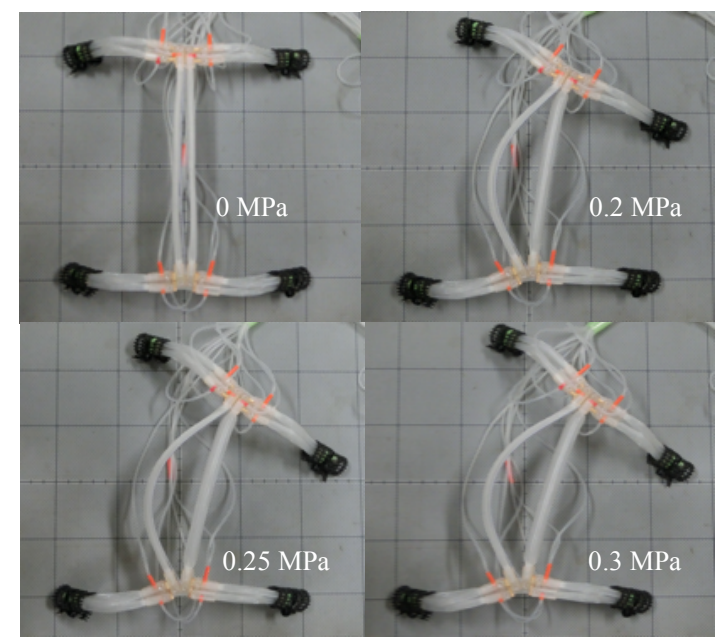

Figure 9. Experimental evaluation of body bending at different input pressure

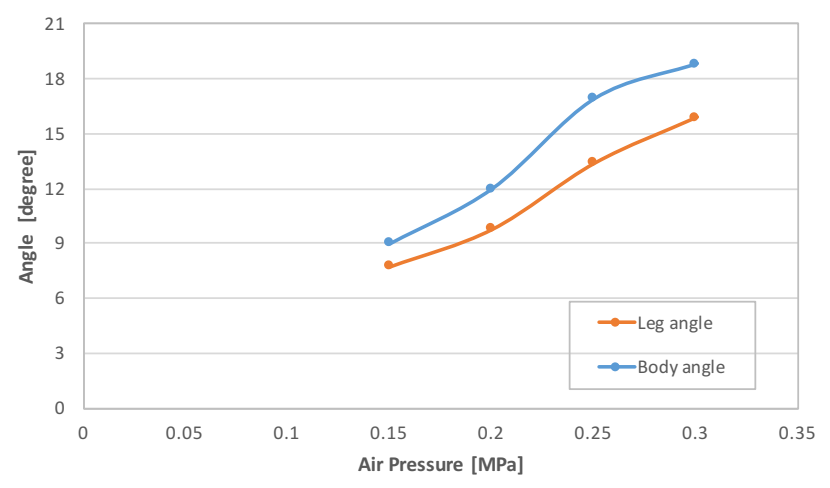

Figure 10. Body and leg angle properties at different input pressure

motion. The gait is divided into only two phases and will be repeated for walking motion as shown in Fig. 11. Leg assignment for the trot gait is shown in Fig. 12 where each leg will be controlled with an on/off valve. The swing and stance phase is regulated to obtain the forward motion. The bending 
motions support the ZMP of the robot for stable walking. During walking, the body may drag along the ground, as in real salamanders locomotion. One cycle of the gait is around $0.5 \mathrm{~s}$ to $1.5 \mathrm{~s}$ depending on the phase frequency. Table III shows the specifications of soft-amphibious gait leg assignment.

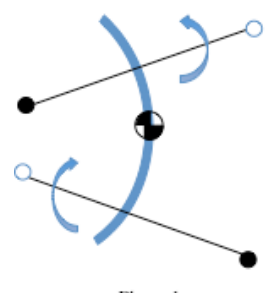

Phase 1

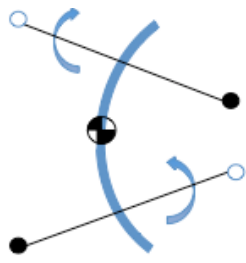

Phase 2
Figure 11. Soft-amphibious robot crawl phases following the salamander locomotion

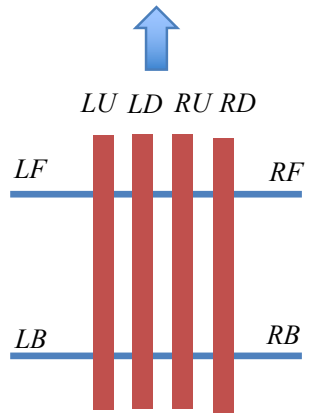

Figure 12. Leg assignment for soft-amphibious robot gait

TABLE III. SPECIFICATION OF SOFT-AMPHIBIOUS ROBOT GAIT LEG ASSIGNMENT

\begin{tabular}{|c|c|c|c|c|c|c|c|c|}
\hline \multirow{2}{*}{ Phase } & \multicolumn{8}{|c|}{ Legs } \\
\hline & $L F$ & $L B$ & $\boldsymbol{R F}$ & $R B$ & $\boldsymbol{L} U$ & $L D$ & $\boldsymbol{R U}$ & $R D$ \\
\hline Phase 1 & 1 & 0 & 0 & 1 & 1 & 1 & 0 & 0 \\
\hline Phase 2 & 0 & 1 & 1 & 0 & 0 & 0 & 1 & 1 \\
\hline \multicolumn{9}{|c|}{$L F=$ left forward } \\
\hline$R F$ & \multicolumn{3}{|c|}{$=$ right forward } & $R B$ & \multicolumn{4}{|c|}{$=$ right backward } \\
\hline$L U$ & \multicolumn{3}{|c|}{$=$ left upper } & $L D$ & \multicolumn{4}{|c|}{$=$ left down } \\
\hline$R U$ & \multicolumn{3}{|c|}{$=$ right upper } & $R D$ & \multicolumn{4}{|c|}{$=$ right down } \\
\hline
\end{tabular}

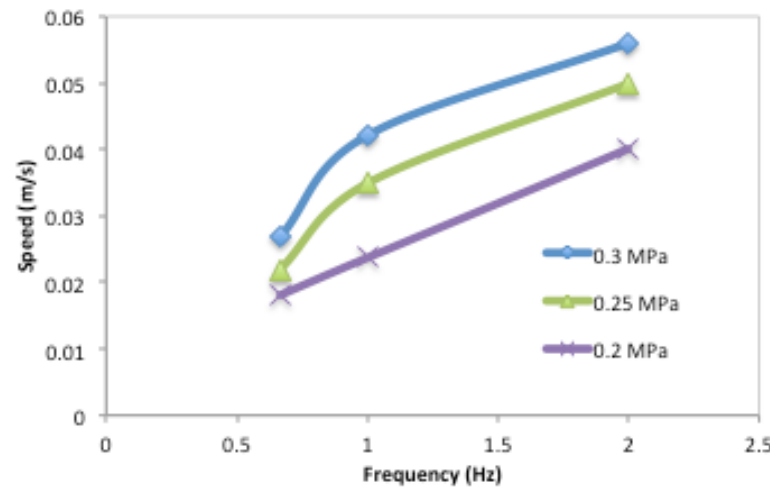

Figure 13. Flat surface walking speed with different frequency and input pressure

For swim motion, the salamander uses an anguilliform swimming gait, which is very similar to the eel locomotion. Swimming is based on axial undulations that are propagated from head to tail along the body. However, for the softamphibious robot developed, we proposed a crawl gait using the leg rotation motion as shown using red arrow in Fig. 7 to replace the anguilliform swimming gait. This gait was also used in WAREC robot for its locomotion [24]. The gait is divided into two phases, the phase of moving legs using the rotational motion and the phase of the moving the body. Anguilliform swim gait was not considered due to limited body length to achieve the travelling wave. As the whole body is lightweight and have buoyancy effect from the actuator used, the robot is able to float during the swim gait.

\section{Walking and swim experiment}

We conducted walking experiment on the soft-amphibious robot to examine the ability of the robot to perform walking motion. For the walking experiment on flat surface, we vary the gait frequency and input pressure as shown in Fig. 13. The result shows that higher input pressure and higher frequency influences the movements for faster motion for the walking speed. Higher pressure will contribute stiffer actuator properties, thus supporting the leg motion for larger stride. At $2 \mathrm{~Hz}$ (fastest frequency), the robot is able to walk with maximum speed of $0.056 \mathrm{~m} / \mathrm{s}$ at $0.3 \mathrm{MPa}$ input pressure.

Fig. 14 shows the walking motion sequence at interval $1 \mathrm{~s}$ for different environment on flat surface, on sand and inside water. The robot successfully walks using trot gait for flat and $10^{\circ}$ incline plane using trot gait. The locomotion on sand and swimming in water environment was performed successfully using crawl gait. Walking on uneven sand surface gives faster speed compared to in water motion as the water contact with the body increases the drag force to move forward. At 0.3 $\mathrm{MPa}$ input pressure and $2 \mathrm{~Hz}$ valve frequencies, the recorded speed was $0.041 \mathrm{~m} / \mathrm{s}$ and $0.022 \mathrm{~m} / \mathrm{s}$, respectively. The experiment also shows that the soft-amphibious robot is robust in dusty, dry and wet environment.

\section{CONCLUSION}

In this paper, a soft-amphibious robot with combination of three actuator links for the leg and four actuators for the body was presented. Structural robot design, walking gait and experiment were also discussed. The robot utilizes two bending characteristics using thin soft McKibben actuator for leg bending and body bending properties. Leg bending properties study was performed in FEM simulation and further validated with experimental test. For body bending property, the deformation of the plastic plate improves the motion for the soft-salamander by supporting the body to mimic the salamander locomotion during walking. The robot succeeded to locomote on flat and incline surface, on sand and in water environment using trot and crawl gaits. Some parameter changes were studied to see the effect of frequency and input pressure on the speed and stability of walking motion. As an extension to the work, it would be interesting to design a longer robot and assess the soft-amphibious robot ability to swim using the Anguilliform swim gait. 
a)

b)

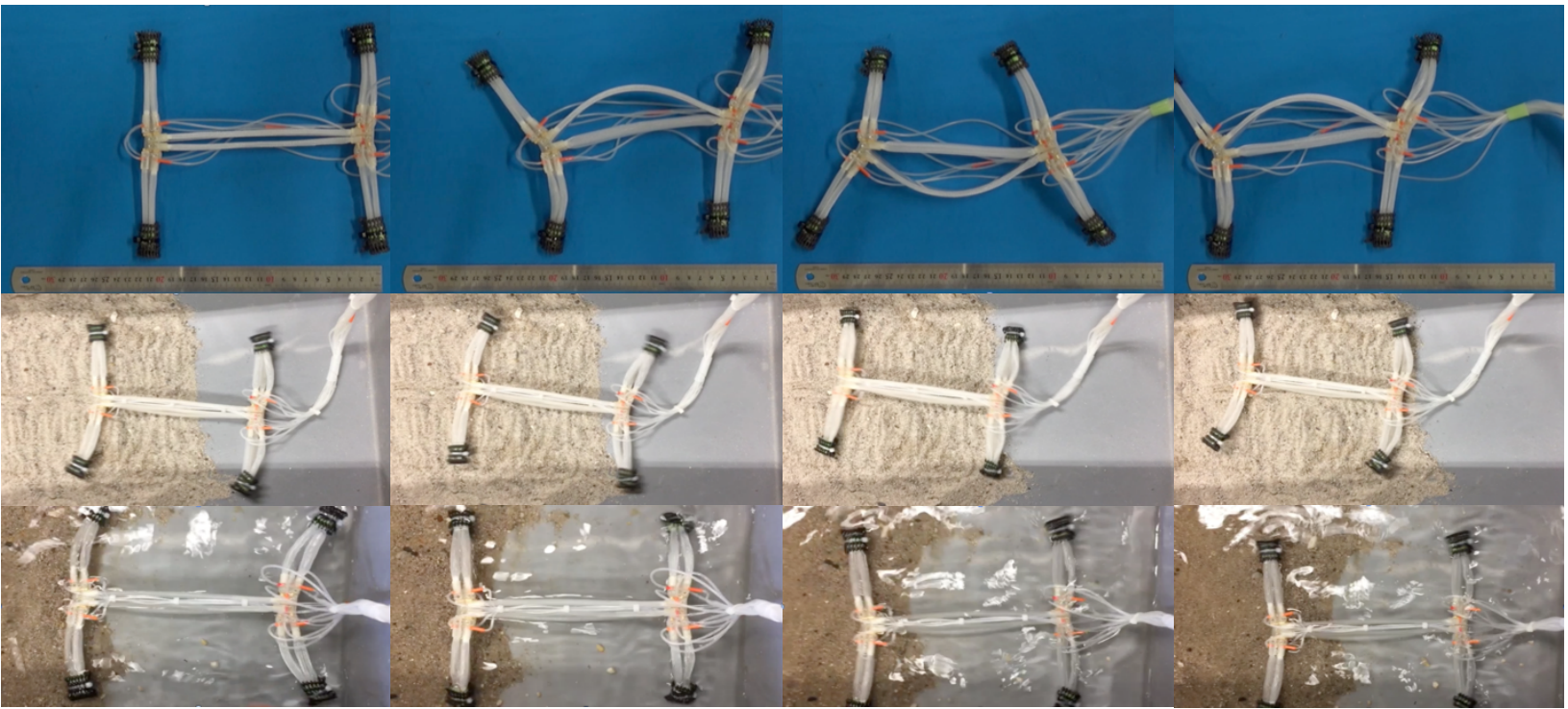

Figure 14. Walking/swimming motion sequence of soft-salamander at interval $1 \mathrm{~s}$ for three different environments

a) on flat surface using trot gait utilizing body bending b) on sand and c) inside water using crawl gait.

\section{REFERENCES}

D. Pongas, M. Mistry, and S. Schaal, "A Robust Quadruped Walking Gait for Traversing Rough Terrain," vol. 307, no. April, pp. 10-14, 2007.

[2] S. Hirose, I. Yoneda, and H. Tsukagoshi, "TITAN VI1 : Quadruped Walking and Manipulating Robot on a Steep Slope," no. April, pp. 494-500, 1997.

[3] I. Conference, "A Miniature Inspection Robot Negotiating Pipes of Widely Varying Diameter," pp. 2735-2740, 2003.

[4] H.-T. Lin, G. G. Leisk, and B. Trimmer, "GoQBot: a caterpillar-inspired soft-bodied rolling robot.," Bioinspir. Biomim., vol. 6 , no. 2, p. 26007, 2011.

[5] A. A. Mohd Faudzi, I. N. A. Mohd Nordin, N. F. Rosli, and M. R. Muhammad Razif, "Application of New Braided Soft Actuator Design in Biomimetic Robot Locomotion," in 15th International Conference on Climbing and Walking Robots and the Support Technologies for Mobile Machines, 2012, pp. 165-172.

[6] M. Calisti, S. Member, A. Arienti, F. Renda, G. Levy, B. Hochner, B. Mazzolai, P. Dario, F. Member, and C. Laschi, "Design and development of a soft robot with crawling and grasping capabilities," pp. 4950-4955, 2012.

[7] A. Crespi, K. Karakasiliotis, and A. J. Ijspeert, "Salamandra Robotica II : An Amphibious Robot to Study Salamander-Like Swimming and Walking Gaits," vol. 29, no. 2, pp. 308-320, 2013.

[8] S. Parasuraman and F. J. Hang, "Statically balanced walking of a crawler robot," Conf. Rec. - IEEE Instrum. Meas. Technol. Conf., pp. $420-425,2011$.

[9] K. Satoshi, "Study of Mechanism and Control of Sprawling-type Quadruped Robot based on Intermittent Trot Gait," Tokyo Institute of Technology, 2016.

[10] K. Hirone, E. Gen, H. Ryuichi, H. Shigeo, and F. F. Edwardo, "How to optimize the slope walking motion by the quadruped walking robot," Adv. Robot., vol. 29, no. 23, pp. 1497-1509, 2015.

[11] F. Giorgio and R. Pieluigia, "Mechanics and simulation of six-legged walking robots," Climbing \&Walking, Towar. New Appl., p. 546, 2007.

[12] R. J. Full and S. T. Michael, "Mechanics of six-legged runners," $J$. Exp. Biol. 148, pp. 129-146, 1990.

[13] C. Kim, H. Shin, and T. Jeong, "Motion Analysis of Lizard Locomotion Using Motion Capture," pp. 2143-2147, 2012.

[14] D. Chang, D. Son, T. Seo, W. Nam, D. Jeon, and J. Kim, "Kinematics-based Gait Planning of a Quadruped Gecko-like Model," pp. 233-238, 2009.

[15] R. M. Alexender, Principles of Animal Locomotion, 2003.
[16] N. Elango, A. A. M. Faudzi, A. Hassan, and M. R. M. Rusydi, "Experimental investigations of skin-like material and computation of its material properties," Int. J. Precis. Eng. Manuf., vol. 15 , no. 9, 2014.

[17] K. Suzumori, S. Wakimoto, K. Miyoshi, and K. Iwata, "Long bending rubber mechanism combined contracting and extending tluidic actuators," IEEE Int. Conf. Intell. Robot. Syst., pp. 44544459, 2013.

[18] I. N. A. M. Nordin, A. A. M. Faudzi, M. R. M. Razif, E. Natarajan, S. Wakimoto, and K. Suzumori, "Simulations of two patterns fiber weaves reinforced in rubber actuator," J. Teknol. (Sciences Eng., vol. 69 , no. 3, 2014.

[19] M. R. M. Razif, A. 'Athif M. Faudzi, M. Bavandi, I. N. A. M. Nordin, E. Natarajan, and O. Yaakob, "Two chambers soft actuator realizing robotic gymnotiform swimmers fin," Robotics and Biomimetics (ROBIO), 2014 IEEE International Conference on. pp. 15-20, 2014.

[20] K. Ogura, S. Wakimoto, K. Suzumori, and Y. Nishioka, "Micro pneumatic curling actuator - Nematode actuator -," in 2008 IEEE International Conference on Robotics and Biomimetics, 2009, pp. 462-467.

[21] S. I. and T. T. M. Takaoka, K. Suzumori, S. Wakimoto, "Fabrication of Thin McKibben Artificial Muscle with Various Design Parameters and Their Experimental Evaluations," Proc. ICMDT2013, p. pp.82, 2013.

[22] H.F Schulte, "The characteristics of the McKibben artificial muscle," Appl. Extern. power Prosthet. Orthetics, vol. 874, pp. 94 $115,1961$.

[23] D. Trivedi, C. D. Rahn, W. M. Kier, and I. D. Walker, "Soft robotics: Biological inspiration, state of the art, and future research," Appl. Bionics Biomech., vol. 5, no. 3, pp. 99-117, Dec. 2008.

[24] T. Matsuzawa, A. Koizumi, K. Hashimoto, X. Sun, and S Hamamoto, "Crawling Gait for Four-limbed Robot and Simulation on Uneven Terrain,” pp. 3-4, 2016. 